\title{
Protein microarray analysis reveals BAFF-binding autoantibodies in systemic lupus erythematosus
}

\author{
Jordan V. Price,,1,2 David J. Haddon,1,2 Dodge Kemmer,1,2 Guillaume Delepine,1,2 \\ Gil Mandelbaum,1,2 Justin A. Jarrell,1,2 Rohit Gupta,,1,2 Imelda Balboni,3 Eliza F. Chakravarty,4 \\ Jeremy Sokolove,, ${ }^{1,5}$ Anthony K. Shum, ${ }^{6}$ Mark S. Anderson, ${ }^{7}$ Mickie H. Cheng, ${ }^{7}$ \\ William H. Robinson,1,5 Sarah K. Browne,8 Steven M. Holland,8 Emily C. Baechler,9 and Paul J. Utz ${ }^{1,2}$ \\ 1Department of Medicine, Division of Immunology and Rheumatology, ${ }^{2}$ Institute for Immunity, Transplantation and Infection (ITI), and \\ 3Department of Pediatrics, Division of Allergy, Immunology and Rheumatology, Stanford University School of Medicine, Stanford, California, USA. \\ ${ }^{4}$ Arthritis and Clinical Immunology, Oklahoma Medical Research Foundation, Oklahoma City, Oklahoma, USA. \\ 5VA Palo Alto Health Care System, Palo Alto, California, USA. ${ }^{6}$ Department of Medicine, Division of Pulmonary and Critical Care, and \\ ${ }^{7}$ Diabetes Center, Department of Medicine, Division of Endocrinology and Metabolism, UCSF, San Francisco, California, USA. \\ ${ }^{8}$ Laboratory of Clinical Infectious Diseases, National Institute of Allergy and Infectious Diseases, NIH, Bethesda, Maryland, USA. \\ ${ }^{9}$ Center for Immunology, University of Minnesota Medical School, Minneapolis, Minnesota, USA.
}

\begin{abstract}
Autoantibodies against cytokines, chemokines, and growth factors inhibit normal immunity and are implicated in inflammatory autoimmune disease and diseases of immune deficiency. In an effort to evaluate serum from autoimmune and immunodeficient patients for Abs against cytokines, chemokines, and growth factors in a high-throughput and unbiased manner, we constructed a multiplex protein microarray for detection of serum factor-binding Abs and used the microarray to detect autoantibody targets in SLE. We designed a nitrocellulosesurface microarray containing human cytokines, chemokines, and other circulating proteins and demonstrated that the array permitted specific detection of serum factor-binding probes. We used the arrays to detect previously described autoantibodies against cytokines in samples from individuals with autoimmune polyendocrine syndrome type 1 and chronic mycobacterial infection. Serum profiling from individuals with SLE revealed that among several targets, elevated IgG autoantibody reactivity to $B$ cell-activating factor (BAFF) was associated with SLE compared with control samples. BAFF reactivity correlated with the severity of disease-associated features, including IFN- $\alpha$-driven SLE pathology. Our results showed that serum factor protein microarrays facilitate detection of autoantibody reactivity to serum factors in human samples and that BAFF-reactive autoantibodies may be associated with an elevated inflammatory disease state within the spectrum of SLE.
\end{abstract}

\section{Introduction}

Abs targeting cytokines, chemokines, and growth factors have been described in SLE, pulmonary alveolar proteinosis, chronic mycobacterial infection, autoimmune polyendocrine syndrome type 1 (APS-1), Felty's syndrome, thymic malignancy, and other immune disorders (1-13). Here, we use the term serum factors to encompass cytokines, chemokines, growth factors, and other circulating components in the blood and lymph that have the ability to influence the immune response. Current methods for the detection of serum factor-reactive Abs have employed several techniques to screen human samples, including Western blot, ELISA, and physiological assays in which reactive sera are used to block the effect or detection of a specific cytokine. Recently, multiplexed detection of serum factor-binding autoantibodies has been achieved using luminescenceand bead-based systems, with the ability to detect Abs from a single sample binding up to 5 cytokine targets in parallel $(11,14)$. While these methods have proven sensitive and useful for target discovery, they have several limitations. Existing techniques are time consuming, require relatively large amounts of patient material (serum or plasma), and - even with the advent of bead-based multiplexing are limited in terms of the number of autoantibody targets that can

Conflict of interest: The authors have declared that no conflict of interest exists. Citation for this article: JClin Invest. 2013;123(12):5135-5145. doi:10.1172/JCI70231. be screened in parallel using a single human sample. We hypothesized that solid-phase protein microarray technology would allow for highly multiplex, comprehensive detection of Abs that bind serum factors. Array-based methods are ideal in that they permit screening of hundreds to thousands of potential $\mathrm{Ab}$ targets using minimal reagents: picogram quantities of analyte and 1-2 microliters of patient serum per assay (15-17). Arrays for the detection of serum proteins associated with immune function have been used in a variety of different contexts and have led to the identification of biomarkers thought to be associated with various diseases states (18-20); however, a high-throughput, multiplexed assay for comprehensive detection of Abs that recognize serum factors has not been developed.

Here we describe a human serum factor protein microarray that could be used to sensitively and specifically detect the reactivity of purified cytokine- and chemokine-reactive mAbs and polyclonal Abs (pAbs), cytokine receptors, and autoantibodies in human patient serum. The platform we developed also included antigens known to be associated with tissue-specific diseases, which allowed us to contextualize serum factor reactivity within a wider profiling of autoantibody reactivity, providing a more complete snapshot of the extent of an individual's autoimmune diathesis.

To demonstrate the power of the array platform to (a) detect previously observed autoantibodies against serum factors and (b) 
A

Probe

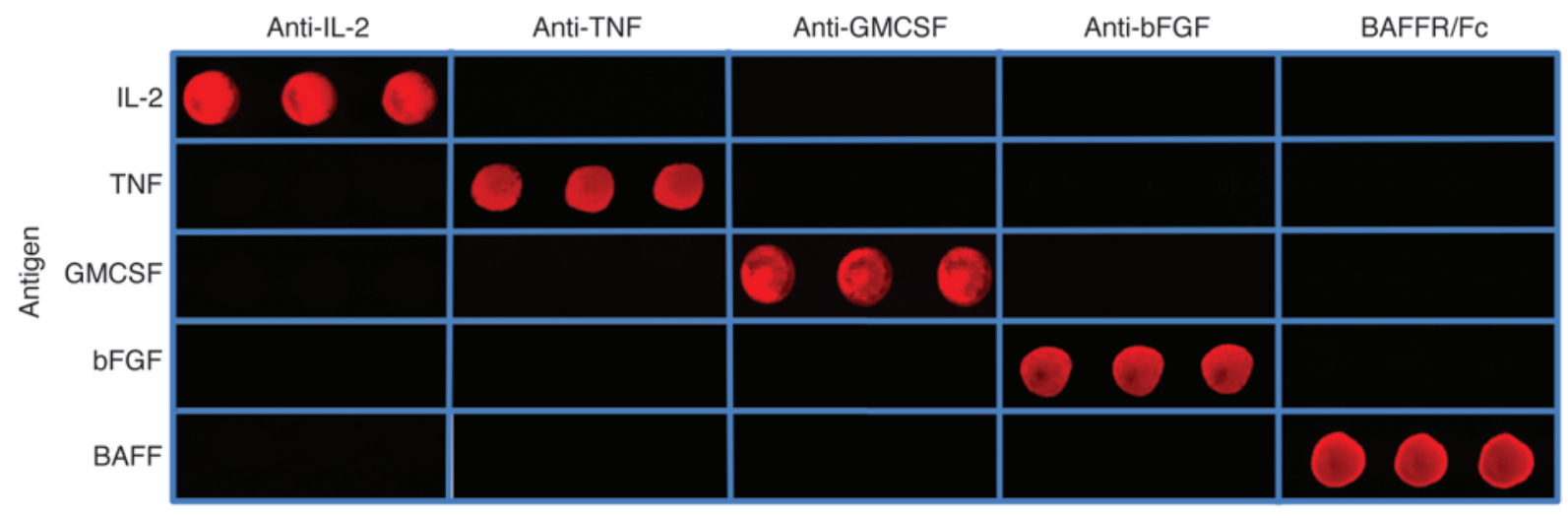

B
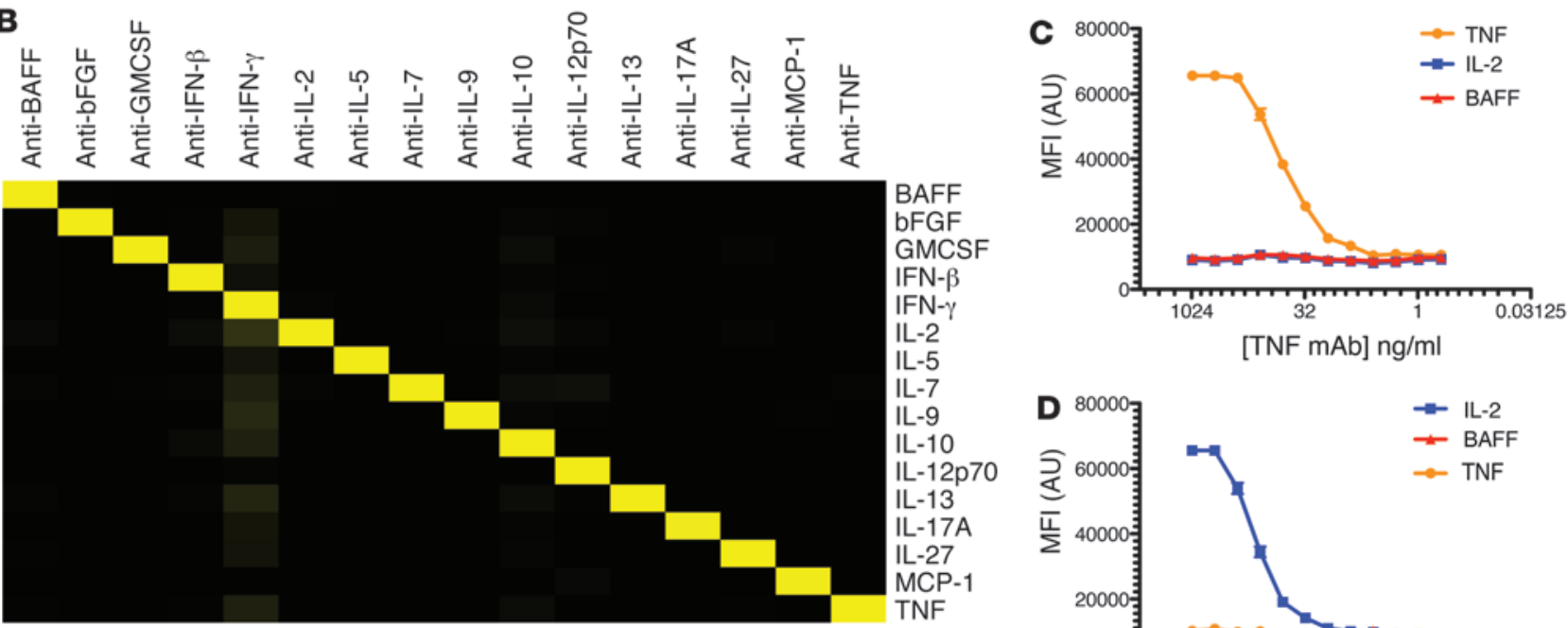

$[\mathrm{TNF} \mathrm{mAb}] \mathrm{ng} / \mathrm{ml}$
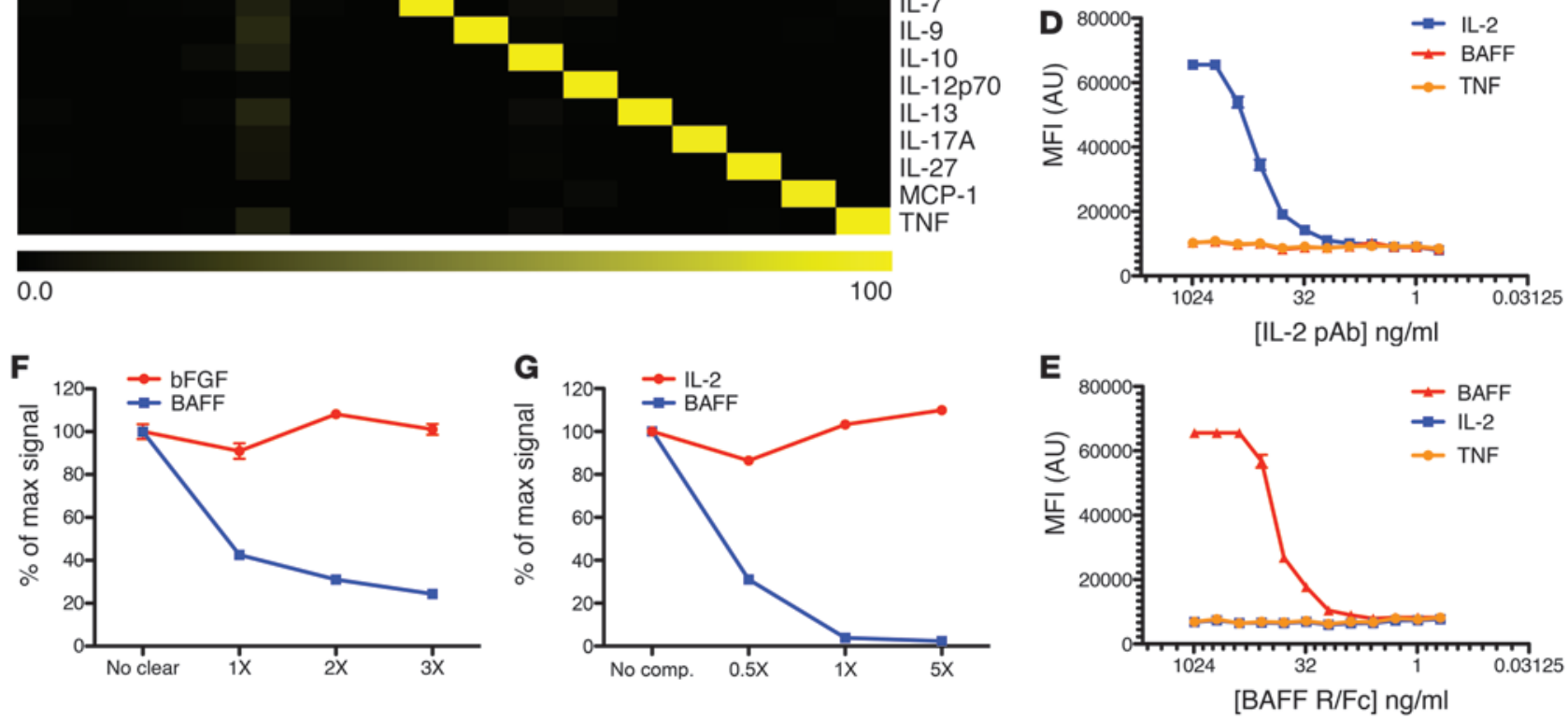

Figure 1

Validation of serum factor array specificity and dynamic range. (A) Qualitative visualization of array results showing the specificity of serum factor-targeted probes for cognate antigen printed in triplicate. 5 separate arrays were probed with Abs against IL-2, TNF, GMCSF, bFGF, and BAFF R/Fc (see Methods and Supplemental Table 1). Reactivity was detected using Cy5-conjugated secondary Abs. Fluorescent images of indicated array features are shown for each probing condition, with each antigen shown in triplicate. (B) Heatmap showing proportion of array-wide maximum MFI at the indicated antigens for arrays probed separately with 16 different Abs. (C-E) Range of detection for reagents (C) TNF mAb, (D) IL-2 pAb, and (E) BAFF R/Fc binding to cognate targets on serum factor microarrays. Data are mean \pm SEM. (F) Array reactivity of a mixture of Abs targeting bFGF and BAFF, either uncleared (No clear) or subjected to 3 rounds of preclearing $(1 \times, 2 \times, 3 \times)$ with BAFF-conjugated beads. Data (mean \pm SEM) are shown as percent maximum MFI signal (relative to uncleared). (G) Array reactivity of a mixture of Abs targeting IL-2 and BAFF, either without competition (No comp) or incubated with $0.5: 1,1: 1$, or $5: 1$ molar ratios of unconjugated BAFF cytokine/Ab $(0.5 \times, 1.0 \times, 5.0 \times$, respectively). Data (mean \pm SEM) are shown as percent maximum MFI signal (relative to no competition). 
contextualize serum factor reactivity within a spectrum of autoantibody reactivity, we profiled samples from 2 cohorts of individuals with immunodeficiency associated with autoreactivity to cytokines: APS-1 and recurrent disseminated mycobacterial infection (DMAC). In validating the array platform's ability to detect cytokine-reactive autoantibodies in human samples, we observed reactivity of IgG autoantibodies against type I IFNs and IL-17 family member cytokines in APS- 1 samples, and to IFN- $\gamma$ in DMAC samples. These results illustrate the potential ability of the serum factor array platform to broadly profile diseases of immune deficiency for the detection of autoimmune recognition of immunomodulatory factors and other targets.

To test the hypothesis that additional autoantibodies against serum factors are present in immune-related diseases, we used the array to screen serum samples from a cohort of individuals with SLE. In addition to identifying autoantibodies targeting welldescribed SLE antigens, we identified novel serum factor-reactive autoantibodies that were associated with SLE compared with healthy controls. Of particular interest were samples with autoantibodies that bound B cell-activating factor of the TNF family (BAFF; also known as BLyS). BAFF is an important molecule in the pathogenesis of SLE and is thought to allow autoreactive $B$ cells to escape negative selection, but also may be involved in more complex interactions involving $\mathrm{T}$ cells and dendritic cells (21-26). In experiments using a BAFF-sensitive reporter cell line expressing the BAFF receptor (27), we identified samples with BAFF-binding autoantibodies that neutralized the signaling activity of BAFF. We observed positive association between BAFF reactivity and high IFN signature, a measure of type I IFN-driven gene expression widely used to characterize SLE subsets (28).

Recently, belimumab, a mAb that neutralizes BAFF, was the first drug approved for the treatment of lupus in more than 40 years (29). Several other BAFF-targeted therapeutics are currently in trials for the treatment of SLE and other inflammatory conditions (30). Given the importance of BAFF in SLE pathogenesis, its status as a treatment target, and the association of antiBAFF autoantibodies with elevated IFN signature and proinflammatory SLE cytokines, it will be important for researchers and clinicians to factor the contribution of naturally occurring BAFFreactive autoantibodies into SLE research and treatment. Furthermore, our present findings highlight the great potential for serum factor microarray analysis to contribute to a deeper understanding of many human autoimmune conditions.

\section{Results}

Cytokine array design, construction, and technical validation. We constructed a nitrocellulose-surface microarray platform containing 59 unique human cytokines and chemokines in addition to 101 autoimmune disease tissue antigens, facilitating the parallel and highly multiplex detection of Abs directed against previously described and novel targets (Supplemental Figure 1 and Supplemental Table 1; supplemental material available online with this article; doi:10.1172/JCI70231DS1). We applied commercial reagents that bind unique cytokine targets to the array to validate the specificity of the platform. Abs directed against IL-2, TNF, GMCSF, and bFGF as well as BAFF receptor/Fc fusion protein $(\mathrm{R} / \mathrm{Fc})$ bound their cognate target on the array and did not crossreact with nonspecific targets (Figure 1A). Across the array, we observed a high degree of specificity using a broad panel of mAbs and pAbs targeting cytokine and chemokine antigens represent- ing diverse structural and functional protein families (Figure 1B). Each probe was specific for its target antigen and displayed low or negligible levels of cross-reactivity to the cognate targets of other probes tested, with the exception of the anti-IFN- $\gamma$ mAb, which displayed cross-reactivity to GM-CSF, IL-2, IL-7, IL-9, IL-10, IL-13, and TNF ranging $10 \%-20 \%$ of the maximum signal (Figure $1 \mathrm{~B}$ and data not shown). The dynamic range of detection on the array was $0.85-1.49 \log _{10}$ for $\mathrm{mAb}, \mathrm{pAb}$, and BAFF R/Fc probes (Figure 1, C-E, and Supplemental Figure 2A). Comparison using ELISA demonstrated that while the array was less sensitive at the low end of Ab concentration, array-based detection was comparable to ELISA for detection of cytokine-binding probes across a wide dynamic range (Supplemental Figure 2B). We verified specific detection of BAFF-targeted commercial reagents on the array in preclearing assays using bead-bound BAFF, and additionally through direct on-array competition with soluble BAFF cytokine. In each case, signal at BAFF array features could be abolished in an antigen-specific manner (Figure 1, F and G). These data indicate that serum factor microarrays allow for the specific detection of serum factor-binding molecules.

Array detection of autoantibodies targeting cytokines in APS-1 and chronic recurrent mycobacterial infection. We next determined whether serum factor microarrays could be used to detect known cytokine-targeted Abs in human samples. To do this, we incubated serum derived from individuals with immunodeficiency diagnoses concomitant with cytokine-targeted Abs. APS- 1 is a disease resulting from deficiency in the autoimmune regulator (AIRE) transcription factor, which controls the education of $\mathrm{T}$ cells to self-antigens during development in the thymus (31). AIRE deficiency results in incomplete negative selection of self-reactive T lymphocytes and systemic autoimmunity (32). Autoantibodies against several cytokine targets have been observed in samples from individuals with APS-1, including Abs targeting type I IFNs and IL-17 family cytokines (e.g., IL-17A, IL-17F, and IL-22; refs. 7-9). We incubated serum from 7 individuals with APS- 1 on the array and compared reactivity with samples from 7 age- and sex-matched healthy control (HC) individuals. In the APS-1 samples, we observed robust reactivity to previously characterized cytokine targets of autoantibodies, including type I IFNs and IL-17 family cytokines (Figure 2A). Additionally, using the significance analysis of microarrays (SAM) algorithm (33), we identified reactivity to several other serum factor and autoimmune tissue antigens that was significantly associated with APS- 1 versus HC samples ( $q<0.001$; Figure $2 \mathrm{~A})$.

Neutralizing autoantibodies against IFN- $\gamma$ have been described by several groups to be associated with chronic recurrent mycobacterial infection and are hypothesized to underlie a deficiency in the Th1-mediated immune response required to control mycobacterial infection (3-6). We tested serum samples from 5 individuals with nontuberculous DMAC that had been previously characterized to have high-titer neutralizing autoantibodies against IFN- $\gamma(5)$. Reactivity to IFN- $\gamma$ was robustly detected in DMAC IFN- $\gamma$-positive samples, compared with 5 negative controls (individuals with clinical mycobacterial infection shown to be negative for anti-IFN- $\gamma$; Figure 2B). These results demonstrated that serum factor arrays can be used to detect cytokine-reactive autoantibodies in human samples and can be used to identify known and potentially novel targets of autoantibodies in diseases of immune deficiency.

Serum factor microarrays reveal autoantibodies against cytokines in a cohort of individuals with SLE. To test whether serum factor arrays could be used to identify known and novel targets of autoanti- 
A
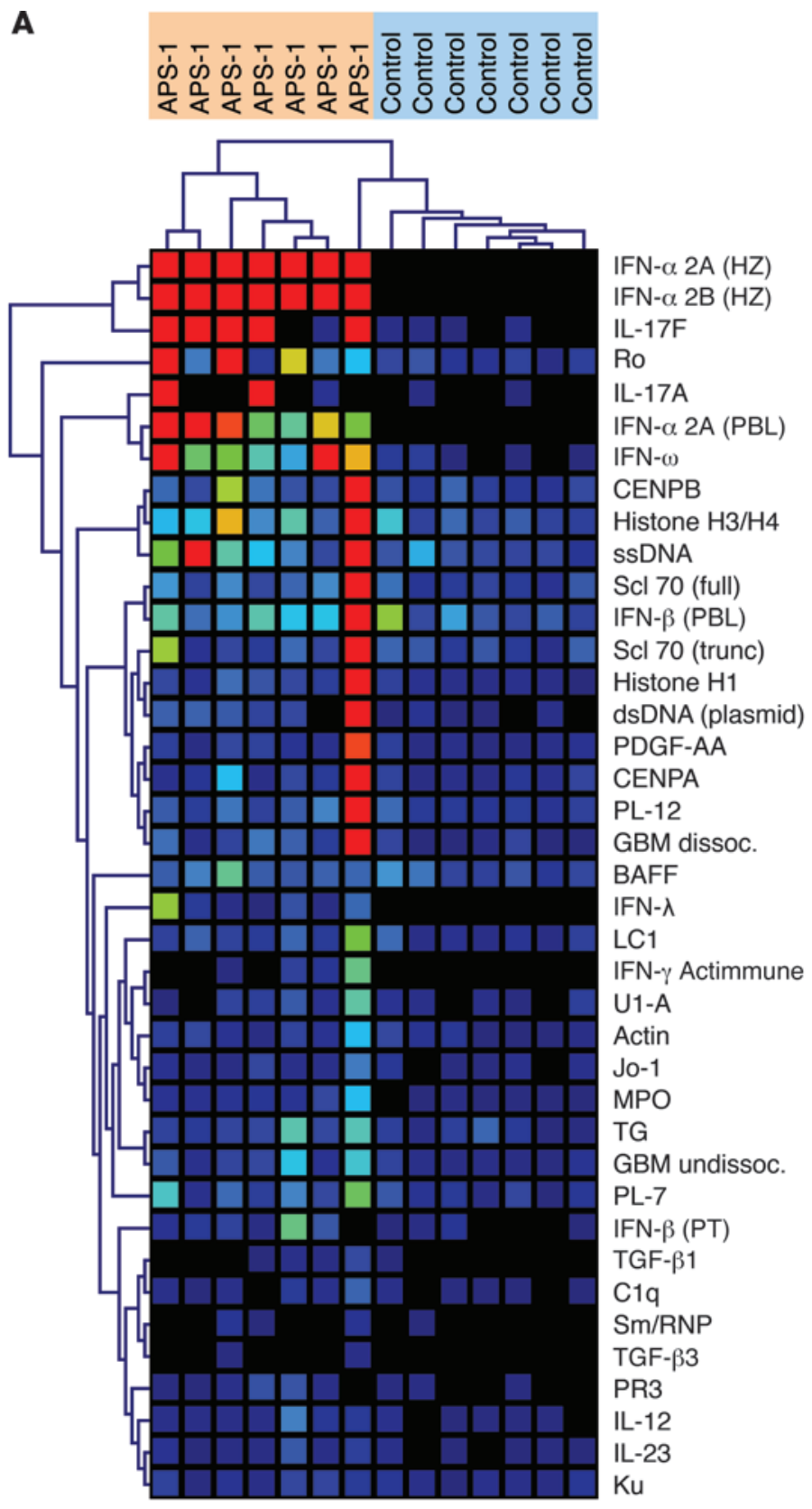

B

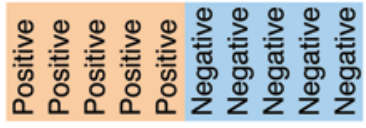

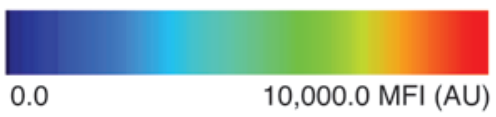

IFN- $\gamma$ Actimmune

IFN- $\gamma$ R\&D

IFN- $\gamma$ eBioscience

Scl 70 (trunc)

Scl 70 (full)

CENPA

Histone $\mathrm{H} 2 \mathrm{a}$

Histone $\mathrm{H} 2 \mathrm{~b}$

Whole histones

PR3

Sm/RNP

IL-12

IL-23

MPO

\section{Figure 2}

Serum factor array analysis of samples from individuals with APS-1 and nontuberculous DMAC. (A and B) Heatmaps displaying serum IgG reactivity significantly associated with (A) APS-1 disease state versus HCs or (B) anti-IFN- $\gamma$-positive nontuberculous DMAC samples versus anti-IFN- $\gamma$-negative mycobacterial infection controls, as assessed by the SAM algorithm by 10,000 permutations of the data $(q<0.001)$.

bodies in individuals with SLE, we screened serum derived from 30 individuals with SLE and 15 HCs associated with the Autoimmune Biomarkers Collaborative Network (ABCoN; ref. 34) cohort (Supplemental Table 2). We observed IgG autoantibody reactivity to several serum factor antigens that was significantly associated with SLE, as assessed using the SAM algorithm $(q<0.001$; Figure 2 and Supplemental Figure 3). Reactivity to serum factor antigens was detected in parallel with reactivity to "hallmark" cell nuclear and connective tissue autoantigens associated with SLE, including RNA- and DNA-associated proteins, connective tissue antigens, and others (Figure 3 and Supplemental Figure 3). Reactivity to nuclear antigens - such as histones, single- and doublestranded DNA, topoisomerase I (Scl-70), and components of the U1 small nuclear ribonucleoprotein RNA splicing complex - as well as connective tissue antigens is well-described in SLE, which indicates that the reactivity we observed to these targets reflects established SLE disease characteristics (35). In addition to these previously described prototypical SLE autoantibody targets, we observed reactivity to several serum factor antigens in sera from individuals with SLE, including BAFF, TGF- $\beta 1-$ TGF- $\beta 3$, IL-2, 
IL-15, IL-23, TNF, IFN- $\alpha 2 \mathrm{~B}$, and IFN- $\gamma$. Additionally, we observed SLE-associated reactivity to EGF and human growth hormone (HGH). These results show that serum factor microarray analysis is a powerful tool for the detection of known and novel targets of autoantibodies in SLE, with the ability to identify reactivity to multiple potentially meaningful array targets in parallel.

Validation of autoantibodies against BAFF and detection in a second cohort of individuals with SLE. To explore the implication of novel targets of autoantibodies identified in our serum factor array analysis, we investigated the potential role of autoantibodies against BAFF observed in the ABCoN SLE cohort. A member of the TNF family of cytokines, BAFF is thought to play a pathologic role in SLE, as high BAFF levels have been observed in SLE patient serum (26), and BAFF has been shown to drive the survival and proliferation of activated $B$ cells with an autoreactive phenotype $(36,37)$. Analysis of the BAFF knockin mouse model revealed a striking autoantibodydriven inflammatory phenotype resembling human SLE, further implicating this molecule as a key factor in SLE disease pathogenesis (22). Recently, a mAb targeting BAFF showed modest efficacy in reducing the frequency and severity of inflammatory flares in SLE, and was approved as the first new drug to treat lupus in more than 40 years $(29,38,39)$. Several other BAFF-targeting drugs are currently in preclinical and clinical development (40).

To validate our array findings and to expand our SLE sample profiling for autoantibodies against BAFF, we performed indirect ELISA on serum samples derived from individuals with SLE and associated HCs in 2 independent cohorts: the $45 \mathrm{ABCoN}$ samples described above, and 93 first-visit samples derived from individuals with SLE enrolled in the Stanford Chronic Immunologic Diseases Registry and Repository (SCIDRR; Supplemental Table 2). In both cohorts, we observed high levels of BAFF reactivity in several SLE patient samples, and while we also observed low to moderate levels of BAFF reactivity in HCs, BAFF reactivity was significantly higher in the SLE groups of the ABCoN and SCIDRR cohorts $(P=0.015$ and $P<0.0001$, respectively; Figure 4$)$. We did not detect a difference in the level of serum BAFF between anti-BAFF-high and -low samples (Supplemental Figure 4).

$B A F F$-reactive sera contain IgG with BAFF-blocking ability. We next tested whether autoantibodies against BAFF have the ability to block BAFF activity by interfering with its ability to signal via the BAFF receptor. To do so, we tested the BAFF-blocking activity of IgG purified from SLE samples using the FL-17 cell line, a BAFF-sensitive mouse myeloma cell line stably transfected with the mouse BAFF receptor fused with a TRAIL endodomain (27). In the presence of human BAFF, FL-17 cells activated apoptosis signaling through caspases 3 and 7 , whose activity we measured with a caspase-dependent luminescence assay, displaying sensitivity within the range of $1.0-250 \mathrm{ng} / \mathrm{ml}$ soluble BAFF (Figure $5 \mathrm{~A}$ ). Addition of soluble BAFF R/Fc, but not soluble TNF R/Fc, was capable of inhibiting the caspase luminescence signal (Figure 5B), which indicated that the FL-17 cell line can be used to measure BAFF-specific inhibition.

Due to the large volume of sera needed to perform BAFF-blocking experiments, we used samples from the SCIDRR cohort to test the ability of autoantibodies binding BAFF to neutralize BAFF signaling via the BAFF receptor. We purified serum IgG from 40 samples -15 each from individuals with SLE that displayed the highest and lowest levels of BAFF binding (anti-BAFF-high and anti-BAFF-low, respectively) and 10 from age- and sex-matched $\mathrm{HCs}$ - and measured the BAFF-blocking ability of the IgG from each sample in the
FL-17 BAFF signaling assay. While there was a slight reduction of caspase signaling in all sample categories compared with the nosample control at the 2 highest sample concentrations across 3 dilutions, IgG from anti-BAFF-high SLE patients blocked BAFF signaling significantly more than anti-BAFF-low and HC IgG $(P<0.001$ for all comparisons; Figure 5C). This indicates that, among patients with high levels of anti-BAFF autoantibodies, there are individuals with IgG Abs capable of blocking BAFF signaling through the BAFF receptor. Interestingly, there was significantly higher IgG-mediated blocking of BAFF signaling in $\mathrm{HC}$ versus anti-BAFF-low samples, which correlates well with the BAFF-binding results measured by ELISA in the same samples (Figure 5C and Supplemental Figure 4). In any given sample, the magnitude of BAFF-blocking activity was related to BAFF binding; however, anti-BAFF-high SLE patient samples displayed differential ability to block the activity of BAFF in the FL-17 cell assay (Figure 5D).

To ensure that the blocking observed in the FL-17 cell assay was specific, we subjected select IgG samples derived from SLE and HC serum to 3 rounds of preclearing with biotinylated BAFF or with biotinylated IL- 2 and IFN- $\gamma$ conjugated to streptavidin-sepharose beads before measuring BAFF-blocking ability. Preclearing with BAFF-bound beads, but not IFN- $\gamma /$ IL-2-bound beads, abrogated the inhibition of caspase signal observed using the BAFF R/Fc control reagent (Figure 5E), which indicated that signal inhibition in the caspase assay is attributable to BAFF-specific neutralizing agents. A similar pattern of abrogated BAFF inhibition was seen in anti-BAFF-high SLE and HC samples (Figure 5, F-H), which indicates that the blocking of BAFF observed in these samples is attributable specifically to Abs that bind BAFF, and not the result of nonspecific interactions. These data indicate that BAFF-binding IgG from a subset of individuals with SLE and HCs neutralizes the activity of BAFF via its interaction with the BAFF receptor.

$S L E$ disease parameters correlate with autoantibody reactivity to BAFF. To determine whether the presence of autoantibodies targeting BAFF was correlated with clinical measures of SLE disease pathology, we analyzed additional data sets associated with samples in the $\mathrm{ABCoN}$ cohort. While the results of this analysis were based on a limited number of samples $(n=30)$ and should be considered preliminary, we observed a connection between the presence of BAFF reactivity and hallmarks of a more severe SLE disease profile. The most striking clinical feature associated with the presence of autoantibodies in the $\mathrm{ABCoN}$ cohort was elevated IFN signature. The IFN signature, a measure of the extent of IFN- $\alpha$-dependent gene transcription in circulating peripheral blood mononuclear cells, has been associated with increased disease severity in individuals with $\operatorname{SLE}(18,41,42)$. The samples we profiled from the $\mathrm{ABCoN}$ cohort were stratified into high and low quartiles of IFN signature, as previously described (18). IFN-high SLE samples contained significantly more reactivity to BAFF - and, indeed, to most of the antigens on the array significantly associated with SLE (Figure 2) - than IFN-low samples ( $q<0.001$; Supplemental Figure 5). Our results are in line with prior studies from our group and others showing that a higher preponderance of autoantibodies against nuclear antigens is associated with a type I IFN signature in $\operatorname{SLE}(17,28)$ and further extend the umbrella of pathology associated with a high IFN signature to include reactivity to serum factor autoantigens.

BAFF reactivity was also significantly positively associated with the levels of a number of serum factor proteins, such as proinflammatory cytokines, chemokines, and cell adhesion factors, including 


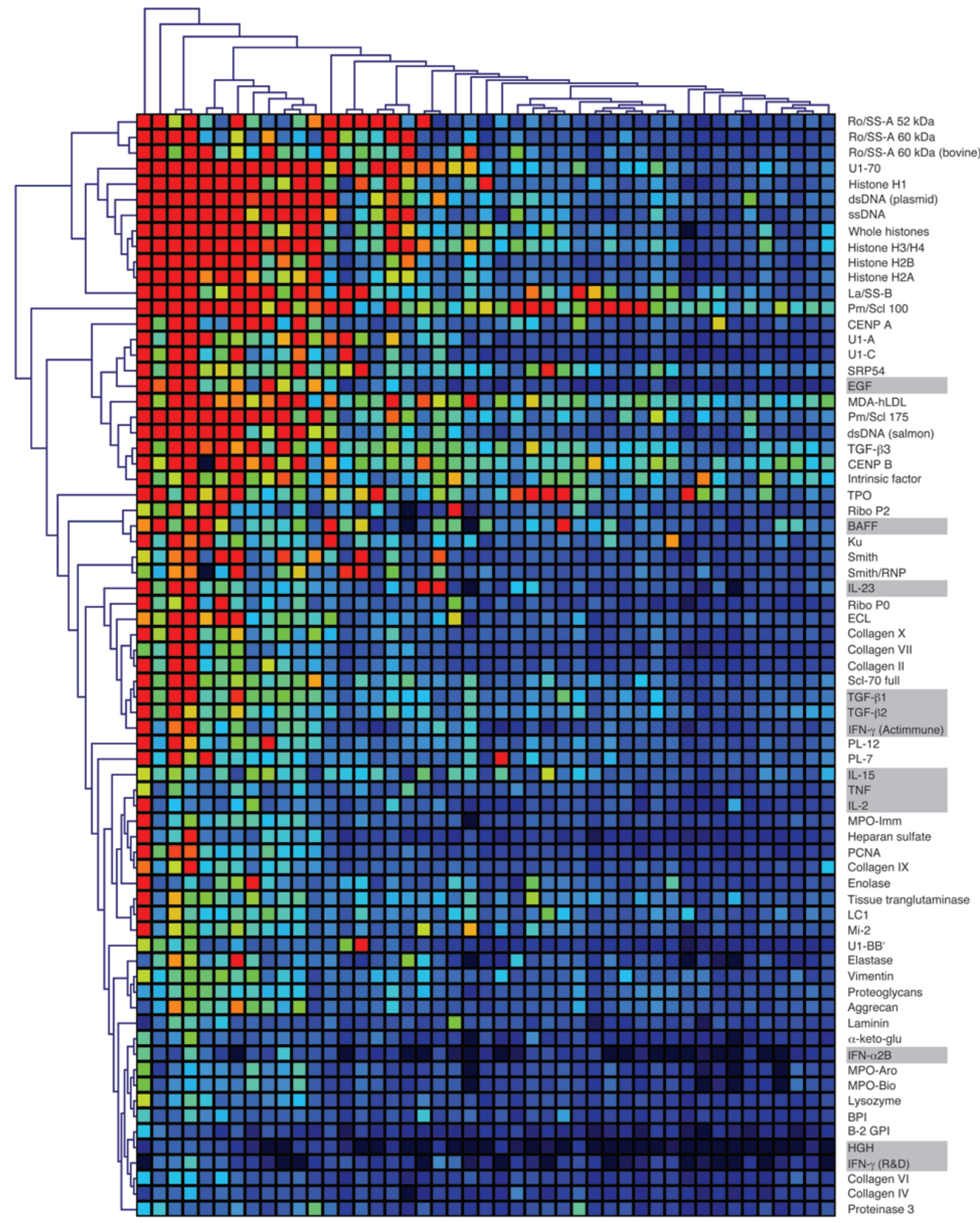




\section{Figure 3}

Serum factor array reactivity in a cohort of individuals with SLE. Heatmap displaying serum IgG reactivity significantly associated with SLE disease state versus HCs, as assessed by the SAM algorithm by 10,000 permutations of the data $(q<0.001)$. Serum factor antigens are shaded gray.

proinflammatory chemokines known to be associated with a high IFN signature in SLE $(P<0.01, q<0.05$; Supplemental Figure 6, Supplemental Table 3, and refs. 18, 43). However, our data indicated that BAFF reactivity was also associated with elevated levels of serum proteins not know to be associated with IFN signature, including IL-1 $\beta$, IL-2, IL-5, TNF, and lymphotoxin, among others $(q<0.05$; Supplemental Figure 6 and Supplemental Table 3); these findings potentially differentiate SLE pathology associated with BAFF reactivity from that associated with high levels of type I IFN-driven gene expression.

\section{Discussion}

It is increasingly clear that autoantibodies targeting cytokines, chemokines, and other circulating immunologic factors can alter and exacerbate the pathology of a variety of immune diseases. We have created a protein microarray platform for rapidly evaluating patient samples for autoantibodies targeting cytokines, chemokines, and other serum factors. The considerable power of serum factor arrays to detect autoantibodies against multiple targets in parallel, using minimal material (on the order of 1-2 $\mu$ l human serum or plasma), makes our platform an ideal tool for screening large numbers of samples in an unbiased manner. To date, the methods used to detect human autoantibodies against serum factors have been relatively low-throughput, and in some cases involved screening large amounts of patient material in living cell assays. While physiological assays involving living cells are an absolute requirement for validation of neutralizing or other immunomodulatory effects of serum factor-targeted autoantibodies, we believe that screening samples for serum factor binding ability on our array platform is currently the most powerful and effective method for autoantibody discovery.

While we focused the present study on SLE, another potentially useful application for the serum factor microarray platform may be the discovery of new targets of autoantibodies in individuals with adult-onset, unexplained immune deficiency, as these patients would fit the criteria for development of autoimmunity targeting one or more critical components of the immune system. Our proof-of-concept data showing the detection of known and novel targets of autoantibodies associated with APS-1 and DMAC demonstrated the potential for discovery of immune factor-targeted autoantibodies that may underlie immune deficiency. We have initiated several projects using serum factor microarrays to test samples from large cohorts of immune-deficient individuals whose disease is not explained by underlying genetic lesions, viruses, or other known causes.

Using the platform to screen samples from individuals with SLE, we identified autoantibodies targeting several serum factors, including previously undescribed reactivity that may factor into the multifaceted inflammatory pathology associated with SLE. We observed elevated autoantibody reactivity to serum factor antigens BAFF, TGF- $\beta 1$-TGF- $\beta 3$, IL-2, IL- 15 , IL- 23 , TNF, IFN$\alpha 2 \mathrm{~B}, \mathrm{IFN}-\gamma, \mathrm{EGF}$, and HGH in SLE patients compared with HCs in the ABCoN cohort. Reactivity to IFN- $\alpha$, a type I IFN, has previously been observed in the serum of individuals with SLE; in one study, IFN- $\alpha$-targeted autoantibodies were shown to block the signaling of IFN- $\alpha$ (1), leading to the hypothesis that neutralizing autoantibodies against IFN- $\alpha$ can ameliorate disease. While direct comparison of the IFN- $\alpha$ reactivity observed by Morimoto et al. (1) with reactivity in our study is not possible because different IFN- $\alpha$ subtypes were used to detect autoantibodies, we also observed that IFN- $\alpha$ autoantibodies were associated with SLE versus HC samples (Figure 3). However, we observed that IFN- $\alpha$ reactivity was associated with a high IFN signature (Supplemental Figure 5). This discrepancy could be explained by differences in the neutralization potential of autoantibodies targeting different IFN- $\alpha$ family members (neutralization of IFN- $\alpha$ was not assessed in our study) or by demographic differences in the cohorts. It will be important to follow up with neutralization studies on the targets of autoantibodies reported herein, to determine whether other serum factorbinding autoantibodies we described have the ability to modulate SLE disease parameters.

Given the importance of BAFF in the pathogenesis of SLE, we chose to investigate the implication of BAFF-binding autoantibodies that we observed to be associated with SLE. We found that BAFF reactivity was linked to IFN signature in the $\mathrm{ABCoN}$ cohort, and there was also partial overlap in the clinical and serum protein correlates that have previously been described for IFN signature, including elevated levels of anti-dsDNA and anti-La autoantibodies and elevated levels of proinflammatory chemokines (18, 41, 43). While our observations were based on a limited number of samples, these results suggest that BAFF reactivity may be one facet of a more severe SLE disease state, with at least partial overlap with increased inflammatory pathology associated with an elevated IFN signature.

Whether BAFF-targeted autoantibodies are a byproduct of a more severe SLE disease state primarily driven by high levels of type I IFNs or serve as a marker of a unique subcohort of individuals with SLE, it is perhaps counterintuitive that autoantibodies binding to and neutralizing a known proinflammatory SLE cytokine were associated with worse disease. Some insight as to the effect of Ab blockade of BAFF in SLE can be gleaned from data associated with the clinical trial of belimumab, the neutralizing $\mathrm{mAb}$ against BAFF recently approved for SLE therapy (29). Belimumab showed only modest efficacy at increasing the time to first SLE flare and decreasing SLE disease parameters, including physician's global assessment and anti-dsDNA titers. The fact that the effects of the drug were modest and not observed in all segments of the study population indicates that the potential effects of BAFF neutralization within the broad spectrum of SLE disease severity are likely to be subtle, and potentially difficult to resolve. Furthermore, our data contribute to a growing body of work showing that BAFFdriven pathology and the effects of BAFF neutralization are likely quite complex and require further study $(39,44)$.

Intriguingly, we observed BAFF-neutralizing autoantibodies in a subset of samples derived from HCs. Cytokine-targeted autoantibodies have been described in healthy human subjects $(45,46)$; however, the implications of this reactivity remain unclear. Small but detectable populations of circulating autoreactive lymphocytes appear in healthy humans, which indicates that immune homeostasis may involve a controlled level of self-recognition 

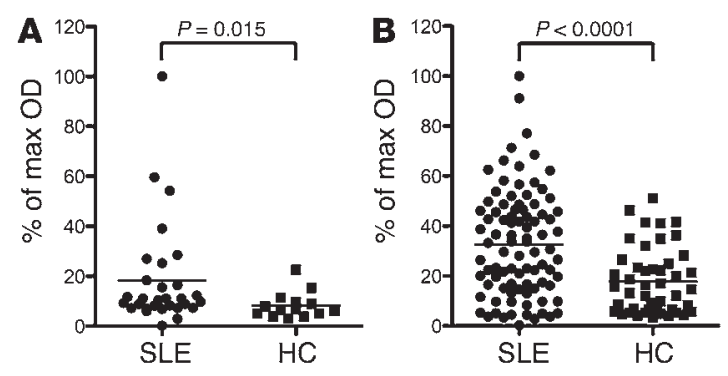

\section{Figure 4}

Detection of BAFF reactivity in 2 independent SLE cohorts by ELISA. (A and B) BAFF reactivity detected in (A) ABCoN and (B) SCIDRR SLE sample cohorts and associated controls (see Supplemental Table 2). Values are presented as a percentage of the sample in each cohort with the highest OD (assigned as 100\%). $P$ values (2-tailed) represent significance of BAFF reactivity association with SLE versus $\mathrm{HC}$ in each cohort, assessed using Mann-Whitney $U$ test.

and autoreactivity (47). The fact that we saw a spectrum of antiBAFF levels across 2 cohorts of samples derived from individuals with SLE in addition to HC samples indicates that autoantibodies against BAFF may be a relatively common occurrence in humans. Higher levels of BAFF protein, known to occur in individuals with SLE, may drive the development of the highly elevated levels of BAFF-binding autoantibodies we observed to be associated with SLE compared with HCs. In studies investigating the development of BAFF-binding autoantibodies that occur in mouse models of SLE, BAFF-targeted autoantibodies increased over time in diseased mice versus controls (our unpublished observations), which indicates that BAFF reactivity develops in tandem with inflammatory pathology associated with SLE. It will be important to investigate the potential triggers of elevated reactivity to serum factor antigens, including BAFF, as researchers continue to piece together the complex web of SLE disease pathology, and as clinicians make treatment decisions that may involve BAFFneutralization therapy.

Of the other novel targets of autoantibodies we identified, the observed reactivity to growth factors EGF and HGH is of particular interest. Further screening of samples derived from individuals with SLE and other inflammatory autoimmune diseases revealed striking array reactivity to these and other growth factor targets, including various isoforms of FGF (data not shown). The idea of an inverse relationship between inflammation- and growth factor-mediated pathways is an area of increasing interest in the field of inflammation (48). The implication of growth factor-targeted autoantibodies in the potential trade-off between tissue growth/repair and inflammation is one intriguing avenue to pursue.

We have shown the usefulness of serum factor microarrays to discover new targets of autoantibodies in human disease. Our platform represents a considerable advance in the field of proteomic analysis of autoantibody-associated immune pathologies, with the potential to make critical discoveries in a spectrum of diseases ranging from immune deficiency to inflammatory autoimmune conditions. One limitation of the arrays in their current format is the high likelihood of undersampling the broad potential autoantibody repertoire directed against serum factor targets. As shown in our validation data (Figure 1 and Supplemental Figure 2), the arrays lacked sensitivity in detection of low-concentration probe, and we may be missing relevant low-abundance or low-affinity autoantibodies. Furthermore, the data reported here reflect the detection of IgG; however, other Ig isotypes may bind to serum factors. To address these potential complications, we are working to incorporate several technological advances developed by our group and our collaborators into the serum factor array platform. We have developed methods that allow for the detection of reactivity on multiple fluorescent channels and may ultimately enable non-fluorescence-based detection $(17,49)$. We have also recently reported the development of array platforms that enhance the level of fluorescence-based detection more than 100-fold (50). Integration of these technologies into the serum factor array platform will allow us to greatly increase the sensitivity of autoantibody detection as well as amplify the amount of information that we can obtain from a single biological sample, which we expect will facilitate more comprehensive studies of the phenomenon of serum factor-targeted autoantibodies.

\section{Methods}

Array design and construction. We printed protein antigens (see Supplemental Table 1) on nitrocellulose-surface glass slides (Whatman) using a VersArray ChipWriter Compact robotic microarrayer and ChipWriter Pro software (BioRad) as described previously (15) in replicates of 3, 4, or 6 across a range of concentration $(0.05-0.2 \mathrm{mg} / \mathrm{ml})$ diluted in PBS (Bio-Rad).

Ab-binding assays. We blocked whole-protein arrays with $5 \% \mathrm{w} / \mathrm{v}$ nonfat milk (BioRad) in PBS for 1.25 hours at room temperature (RT) with light rocking agitation. After rinsing arrays 3 times with sample buffer (PBS plus $0.25 \%$ Tween-20 [Sigma-Aldrich] [PBST] with 2.5\% FCS [Omega Scientific]), we applied the indicated commercial Abs (anti-BAFF pAb, catalog no. ab5965, Abcam; anti-bFGF mAb, catalog no. 610072, BD Biosciences; anti-GMCSF mAb, catalog no. 554502, BD; anti-IFN- $\beta$ pAb, catalog no. ab84258, Abcam; anti-IFN- $\gamma$ mAb, catalog no. 14-7317-81, eBioscience; anti-IL-2 pAb, catalog no. 13-7028-81, eBioscience; anti-IL-5 $\mathrm{mAb}$, catalog no. 14-7072-85, eBioscience; anti-IL-7 mAb, catalog no. 501301, Biolegend; anti-IL-9 mAb, catalog no. 558088, BD; anti-IL-10 $\mathrm{mAb}$, catalog no. 14-7108-81, eBioscience; anti-IL-12p70 mAb, catalog no. 3450-8-250, Mabtech; anti-IL-13 mAb, catalog no. 14-7139-81, eBioscience; anti-IL-17A mAb, catalog no. 14-7178-81, eBioscience; anti-IL-27 $\mathrm{mAb}$, catalog no. AF2526, R\&D Systems; anti-MCP-1 mAb, catalog no. 14-7099-81, eBioscience; anti-TNF mAb, catalog no. 14-7348-81, eBioscience) diluted to $1.0 \mu \mathrm{g} / \mathrm{ml}$, BAFF R/Fc (catalog no. 1162-BR-050, R\&D) diluted to $1.0 \mu \mathrm{g} / \mathrm{ml}$, or patient serum diluted 1:250 in sample buffer for 1.25 hours at $4^{\circ} \mathrm{C}$ with light rocking agitation. We then rinsed the arrays 3 times followed by 35 -minute washes in sample buffer before applying secondary $\mathrm{Ab}$ reagents (goat anti-mouse IgG, goat anti-rabbit IgG, goat anti-human IgG, goat anti-rat IgG, or donkey anti-goat IgG conjugated to Cy5, Jackson ImmunoResearch) diluted to $0.375 \mu \mathrm{g} / \mathrm{ml}$ in sample buffer. After incubation of detection $\mathrm{Ab}$ for 45 minutes at $4^{\circ} \mathrm{C}$ with light rocking agitation, we rinsed arrays 3 times followed by 35 -minute washes in sample buffer, then rinsed arrays in PBS followed by deionized $\mathrm{H}_{2} \mathrm{O}$. We dried arrays in microscope slide racks centrifuged at $300 \mathrm{~g}$ for 5 minutes at RT.

Indirect BAFF ELISA. We coated Nunc-Immuno Maxisorp 96-well plates (Thermo Scientific) overnight at $4^{\circ} \mathrm{C}$ with BAFF (R\&D) at $1 \mu \mathrm{g} / \mathrm{ml}$ diluted in carbonate buffer ( $\mathrm{pH}$ 9.5). For probing, we washed coated plates 3 times with PBST and then blocked with PBST plus 30\% FCS for 30 minutes at RT. We then washed plates 3 times in PBST and applied patient serum diluted 1:1,200 in PBST plus 30\% FCS in triplicate in a volume of $50 \mu \mathrm{l} /$ well for 2 hours at RT. After washing plates 5 times with PBST, we applied peroxidase-conjugated goat anti-human IgG (heavy and light chain) secondary Abs (Jackson ImmunoResearch) diluted to $0.16 \mu \mathrm{g} / \mathrm{ml}$ 
A
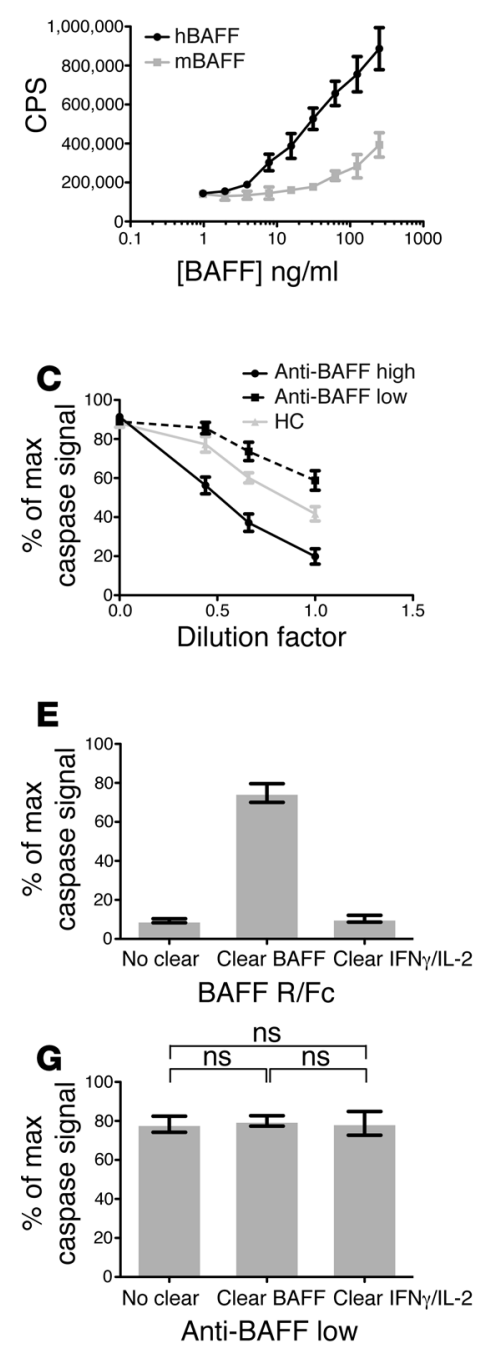

B

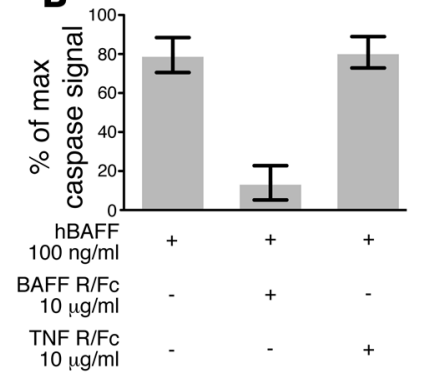

D
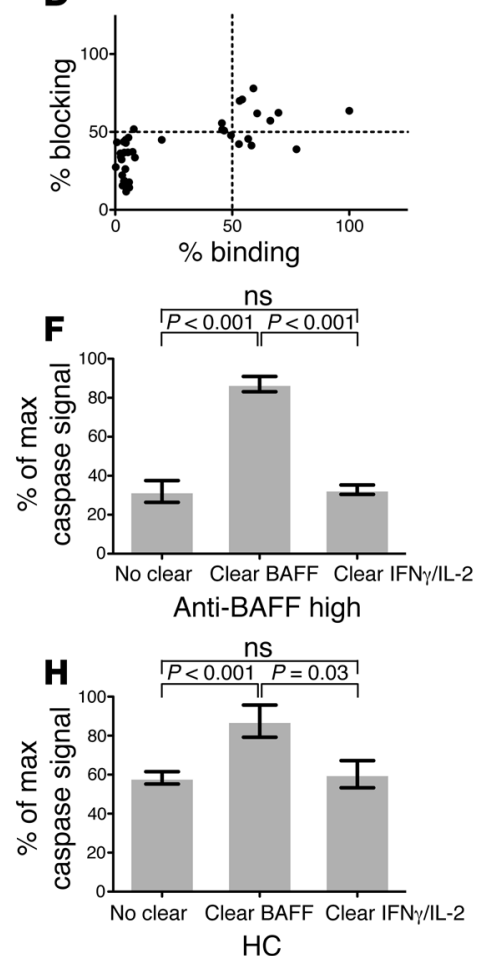

\section{Figure 5}

Detection of BAFF blocking activity in SLE and HC samples using FL-17 cells. (A) Caspase luminescence signal in wells containing FL-17 cells incubated with a serial dilution of soluble recombinant human and mouse BAFF (hBAFF and mBAFF, respectively), showing a sensitivity range of $1.0-250.0 \mathrm{ng} / \mathrm{ml}$. CPS, counts per second. (B) Luminescent signal of FL-17 cells incubated with recombinant human BAFF in the presence of no inhibitor, BAFF R/Fc, or TNF R/Fc. (C) Inhibition of luminescence signal of $\mathrm{FL}-17$ cells incubated with $100 \mathrm{ng} / \mathrm{ml}$ recombinant human BAFF in the presence of a serial dilution of IgG purified from individual anti-BAFF-high $(n=15)$, antiBAFF-low $(n=15)$, and $\mathrm{HC}(n=10)$ serum samples. $P<0.001$ for all between-group comparisons, 2-way ANOVA and Tukey correction for multiple comparisons. (A-C) Data represent mean \pm SEM of $\geq 4$ replicate wells. (D) Relationship between BAFF binding and BAFF blocking level in each of 40 SCIDRR cohort samples in the FL-17 assay. Blocking percentage was calculated as average CPS per sample normalized to maximum signal (no sample condition). (E-H) Caspase luminescence signal in FL-17 cells incubated with $100 \mathrm{ng} / \mathrm{ml} \mathrm{BAFF}$ in

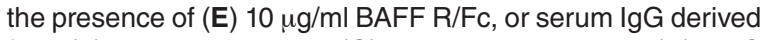
from (F) 6 anti-BAFF-high, (G) 4 anti-BAFF-low, and (H) 4 HC samples from the SCIDRR cohort, with or without preclearing with sepharose beads conjugated to BAFF or IFN- $\gamma /$ IL-2. Data are mean \pm SEM. $P$ values (2-tailed) were calculated by Mann-Whitney $U$ test. in PBST plus 30\% FCS for 1 hour at RT. We then washed plates 5 times in PBST and added TMB One-Step Substrate (Dako) for 5-15 minutes prior to terminating the reaction with $2 \mathrm{M}$ sulfuric acid (Sigma-Aldrich). We obtained colorimetric readings using a SpectraMAX 190 plate reader ( $\lambda=450$; Molecular Devices).

Serum protein profiling. We surveyed serum proteins from the $\mathrm{ABCoN}$ cohort as previously described (18). We measured BAFF serum concentration using a Quantikine ELISA kit (R\&D) according to the manufacturer's instructions.

Protein A/G purification of serum IgG. We purified IgG from $100 \mu \mathrm{l}$ human serum using $0.2 \mathrm{ml} \mathrm{NAb}$ protein $\mathrm{A} / \mathrm{G}$ spin columns (Thermo Scientific) according to the manufacturer's instructions. Eluted fractions from each individual sample were pooled and maintained at $4{ }^{\circ} \mathrm{C}$ in elution/neutralization (EN) buffer for use in FL-17 cell BAFF activity assay.

FL-17 cell BAFF activity assay. FL-17 cells (gift from A. Meager, National Institute for Biological Standards and Control, Hertfordshire, United Kingdom) were maintained as described previously (27). For BAFF stimulation experiments, we seeded 384-well plates (Corning) with $5.0 \times 10^{3}$ cells/well at a volume of $25 \mu \mathrm{l} /$ well, and incubated plates overnight at $37^{\circ} \mathrm{C}$. We diluted BAFF, BAFF R/Fc, TNF R/Fc (R\&D Systems), and IgG purified from human serum samples in sterile-filtered EN buffer (1:20 ratio of Pierce Nab neutralization/elution buffer supplemented with $2 \%$ FCS) and incubated stimulation reagents on FL-17 cells in a volume of $25 \mu \mathrm{l} /$ well with a minimum of 4 wells/condition for 4 hours at $37^{\circ} \mathrm{C}$. After stimulation, we applied Caspase Glo luminescence reagent (Promega), diluted 1:3 in Glo Lysis buffer (Promega), to the cells in a volume of $25 \mu \mathrm{l} /$ well. After 15 minutes of incubation, we measured luminescent signal using a Tecan Infinite M1000 plate reader (Tecan).

Preclearing and competition assay. For preclearing experiments, we diluted BAFF $(0.1 \mathrm{mg} / \mathrm{ml})$ or a mixture of IL- 2 and IFN- $\gamma(0.1 \mathrm{mg} / \mathrm{ml}$ each $)$ in PBS for biotinylation using Chromalink Sulfo-NHS biotin (Solulink) according to the manufacturer's instructions. After passing biotinylated cytokine 2 times through $0.5 \mathrm{ml}$ Zeba desalting columns (Thermo Scientific), we incubated biotinylated cytokines at $0.1 \mathrm{mg} / \mathrm{ml}$ with streptavidinsepharose beads (GE Healthcare) for 30 minutes at RT. We then washed cytokine-bound beads twice in PBS plus 10\% FCS and then added beads either to indicated commercial Abs diluted to $1.0 \mu \mathrm{g} / \mathrm{ml}$ in sample buffer (for array preclearing experiments) or IgG purified from human serum diluted in EN buffer (for FL-17 preclearing experiments) for 20 minutes at RT. After incubation, we centrifuged samples at $850 \mathrm{~g}$ for 1 minute to pellet $\mathrm{Ab} /$ cytokine bead complexes and transferred the supernatant to a new tube. We repeated preclearing of each sample 3 times prior to incubation on serum factor arrays or for use in the FL-17 cell BAFF activity assay. We 
performed competition assays using the conditions described above for the Ab-binding assay with the addition of soluble BAFF corresponding to $0.5: 1,1: 1$, and $5: 1$ molar ratios to $0.05 \mu \mathrm{g} / \mathrm{ml} \mathrm{pAb}$ targeting BAFF (Abcam) during the primary incubation step.

Sources of human samples and storage. APS-1, DMAC, and control samples were provided by authors affiliated with UCSF and NIH. Serum samples from SLE patients, collected as part of $\mathrm{ABCoN}$, were maintained at $-80^{\circ} \mathrm{C}$. Gene expression of peripheral blood mononuclear cells had been previously analyzed and was used to assign IFN-responsive gene signatures, as described previously $(18,41)$. Whole blood was collected from individuals with rheumatologic diseases and $\mathrm{HC}$ volunteers (18 to $\geq 89$ years of age) enrolled in the Stanford University Immunological and Rheumatic Disease Database: Disease Activity and Biomarker Study. We collected $\geq 100 \mathrm{cc}$ whole blood from each individual and allowed the blood to coagulate for 30 minutes after blood draw time. We then centrifuged Vacutainer SST Blood Collection Tubes (BD) at $887 \mathrm{~g}$ for 10 minutes. After centrifugation, we stored the serum layer in $500 \mu \mathrm{l}$ aliquots at $-80^{\circ} \mathrm{C}$.

Statistics. For comparison of array and ELISA dynamic range, we calculated the limit of blank (LOB), limit of detection (LOD), and limit of quantitation (LOQ) for both array and ELISA detection of TNF mAb, IL-2 pAb, and BAFF R/Fc probes according to standard procedures (51). We calculated the high end of the dynamic range of the signal (limit of linearity [LOL]) by determining the $95 \%$ confidence interval of a linear regression weighted to the $\mathrm{EC}_{50}$ of binding curve for each individual probe, determining the highest concentration point that fell within this range. For array reactivity (Figures 2 and 3 and Supplemental Figures 3 and 5), we applied the SAM algorithm to normalized array median fluorescence intensity (MFI) values. For normalization, we divided mean array feature MFI minus local background (MFI-B) by the MFI-B recorded at a human IgG secondary Ab control feature printed on each array, and then multiplied by a scaling factor of 10,000 . We determined reactivity to be significantly correlated with SLE (vs. HC) or IFN-high SLE (vs. IFN-low SLE) by $\geq 10,000$ permutations of repeated measurements, with a false discovery rate of $0(q<0.001)$. For comparison of BAFF reactivity based on clinical features of SLE disease and disease history, we grouped patients based on clinical variables and compared groups using Fisher's exact test. To account for multiple comparisons, we randomly permuted the group labels for each clinical variable 1,000 times and used the distribution $P$ values to calculate estimated $P$ values using the "permute" package of R (www.r-project.org). For correlation of BAFF reactivity with measurements of serum proteins, we performed linear regression analysis and calculated $P$ values associated with the Spearman $r$ for each variable and MFI values of BAFF reactivity measured by serum factor microarray. We corrected for testing of multiple hypotheses using the Benjamini and Hochberg method to generate $q$ values. We generated binding reactivity heatmaps using Multiexperiment Viewer (MEV TM4 Microarray Software Suite, version 10.2; Dana-Farber Cancer Institute) using average linkage Euclidean distance hierarchical clustering.
Study approval. APS-1, DMAC, and control samples were collected with IRB approval from UCSF (protocol no. 10-02467) and the National Institute of Allergy and Infectious Diseases (protocol nos. 93-I-0119 and 02-I-0202). Approval for use of the $\mathrm{ABCoN}$ samples was granted by the University of Minnesota IRB (protocol no. 0110M09982). The study of individuals enrolled the Stanford University Immunological and Rheumatic Disease Database: Disease Activity and Biomarker Study was approved in 2012 by the IRB of the Research Compliance Office at Stanford University (protocol no. 14734). Written informed consent was obtained from all subjects in the study.

Accession numbers. Microarray data were deposited in GEO, with accession nos. GSE51101 and GSE51102 for data in Figure 2 and accession no. GSE51100 for data in Figure 3.

\section{Acknowledgments}

We thank E. Munoz and L. Tarter for coordination with the Stanford Chronic Immunologic Disease Registry and Repository and A. Meager for providing the FL-17 BAFF reporter cell line. J.V. Price was funded by a US National Science Foundation Graduate Research Fellowship (NSF GRFP) and was supported by the Stanford Genome Training Program (SGTP; NIH, US National Human Genome Research Institute). D.J. Haddon was funded by a Canadian Institutes of Health Research (CIHR) postdoctoral fellowship. A.K. Shum, M.H. Cheng, and M.S. Anderson were funded by the National Organization for Rare Disorders. I. Balboni is supported by NIH grant K08 AI080945. P.J. Utz is the recipient of a Donald E. and Delia B. Baxter Foundation Career Development Award, a gift from the Floren Family Trust, and a gift from the Ben May Charitable Trust of Mobile, Alabama, and is supported by NHLBI Proteomics Contract HHSN288201000034C, Proteomics of Inflammatory Immunity and Pulmonary Arterial Hypertension, NIH (grant nos. 5 U19AI082719 and 1 U19-AI090019), Alliance for Lupus Research (grant no. 21858), Canadian Institutes of Health Research (grant no. OR-92141), Stanford Institute for Immunity, Transplantation, and Infection (ITI) Pilot Grant, and FP grant no. 261. The research leading to these results has received funding from the European Union Seventh Framework Programme (FP7/20072013) under grant agreement no. 261382.

Received for publication April 1, 2013, and accepted in revised form September 3, 2013.

Address correspondence to: Jordan V. Price or Paul J. Utz, Division of Immunology and Rheumatology, Stanford University School of Medicine, CCSR Building, Room 2215, Stanford, California 94305, USA. Phone: 650.724.5421; Fax: 650.723.7509; E-mail: jvp2099@gmail.com (J.V. Price); pjutz@stanford.edu (P.J. Utz).
1. Morimoto AM, et al. Association of endogenous antiinterferon- $\alpha$ autoantibodies with decreased interferon-pathway and disease activity in patients with systemic lupus erythematosus. Arthritis Rheum. 2011; 63(8):2407-2415

2. Uchida K, et al. GM-CSF autoantibodies and neutrophil dysfunction in pulmonary alveolar proteinosis. NEngl J Med. 2007;356(6):567-579.

3. Doffinger R, et al. Autoantibodies to interferongamma in a patient with selective susceptibility to mycobacterial infection and organ-specific autoimmunity. Clin Infect Dis. 2004;38(1):e10-e14.

4. Hoflich C, et al. Naturally occurring anti-IFNgamma autoantibody and severe infections with Mycobacterium cheloneae and Burkholderia cocovenenans. Blood. 2004;103(2):673-675.
5. Patel SY, et al. Anti-IFN- $\gamma$ autoantibodies in disseminated nontuberculous mycobacterial infections. J Immunol. 2005;175(7):4769-4776.

6 . Browne SK, et al. Adult-onset immunodeficiency in Thailand and Taiwan. N Engl J Med. 2012; 367(8):725-734.

7. Meager A, et al. Anti-interferon autoantibodies in autoimmune polyendocrinopathy syndrome type 1 . PLoS Med. 2006;3(7):e289.

8. Puel A, et al. Autoantibodies against IL-17A, IL-17F and IL-22 in patients with chronic mucocutaneous candidiasis and autoimmune polyendocrine syndrome type I. J Exp Med. 2010;207(2):291-297.

9. Kisand K, et al. Chronic mucocutaneous candidiasis in APECED or thymoma patients correlates with autoimmunity to Th17-associated cytokines.
JExp Med. 2010;207(2):299-308.

10. Hellmich B, Csernok E, Schatz H, Gross WL, Schnabel A. Autoantibodies against granulocyte colonystimulating factor in Felty's syndrome and neutropenic systemic lupus erythematosus. Arthritis Rheum. 2002;46(9):2384-2391.

11. Burbelo PD, et al. Anti-cytokine autoantibodies are associated with opportunistic infection in patients with thymic neoplasia. Blood. 2010; 116(23):4848-4858.

12. Browne SK, Holland SM. Anticytokine autoantibodies in infectious diseases: pathogenesis and mechanisms. Lancet Infect Dis. 2010;10(12):875-885.

13. Browne SK, Holland SM. Immunodeficiency secondary to anticytokine autoantibodies. Curr Opin Allergy Clin Immunol. 2010;10(6):534-541. 
14. Ding L, Mo A, Jutivorakool K, Pancholi M, Holland SM, Browne SK. Determination of human anticytokine autoantibody profiles using a particle-based approach. J Clin Immunol. 2012;32(2):238-245.

15. Robinson WH, et al. Autoantigen microarrays for multiplex characterization of autoantibody responses. Nat Med. 2002;8(3):295-301.

16. Hueber W, et al. Antigen microarray profiling of autoantibodies in rheumatoid arthritis. Arthritis Rheum. 2005;52(9):2645-2655.

17. Price JV, et al. On silico peptide microarrays for high-resolution mapping of antibody epitopes and diverse protein-protein interactions. Nat Med. 2012; 18(9):1434-1440.

18. Bauer JW, et al. Elevated serum levels of interferon-regulated chemokines are biomarkers for active human systemic lupus erythematosus. PLoS Med. 2006; 3(12):2274-2284

19. Ray S, et al. Classification and prediction of clinical Alzheimer's diagnosis based on plasma signaling proteins. Nat Med. 2007;13(11):1359-1362.

20. Britschgi M, Wyss-Coray T. Blood protein signature for the early diagnosis of Alzheimer disease. Arch Neurol. 2009;66(2):161-165.

21. Hilbert DM, et al. BLyS, a novel member of the tumor necrosis factor family and potent B lymphocyte stimulator. Blood. 1999;94(2):584A-584A.

22. Mackay F, et al. Mice transgenic for BAFF develop lymphocytic disorders along with autoimmune manifestations. J Exp Med. 1999; 190(11):1697-1710.

23. Stohl W. SLE - systemic lupus erythematosus: a BLySful, yet BAFFling, disorder. Arthritis Res Ther. 2003; 5(3):136-138.

24. Lesley R, et al. Reduced competitiveness of autoantigen-engaged $\mathrm{B}$ cells due to increased dependence on BAFF. Immunity. 2004;20(4):441-453.

25 . Thien $M$, et al. Excess BAFF rescues self-reactive $B$ cells from peripheral deletion and allows them to enter forbidden follicular and marginal zone niches. Immunity. 2004;20(6):785-798.

26. Pers JO, et al. BAFF overexpression is associated with autoantibody production in autoimmune diseases. Ann N Y Acad Sci. 2005;1050:34-39.

27. McClements M, Williams S, Ball C, Bristow A, Wadhwa M, Meager A. A novel bioassay for B-cell activating factor (BAFF) based on expression of a BAFF-receptor ectodomain-tumour necrosis factor-related apoptosis-inducing ligand (TRAIL) receptor- 2 endodomain fusion receptor in human rhabdomyosarcoma cells. J Immunol Methods. 2008; 337(1):63-70.

28. Obermoser G, Pascual V. The interferon-alpha signature of systemic lupus erythematosus. Lupus. 2010; 19(9):1012-1019.

29. Wallace DJ, et al. A phase II, randomized, doubleblind, placebo-controlled, dose-ranging study of belimumab in patients with active systemic lupus erythematosus. Arthritis Rheum. 2009;61(9):1168-1178.

30. Fairfax K, Mackay IR, Mackay F. BAFF/BLyS inhibitors: A new prospect for treatment of systemic lupus erythematosus. IUBMB Life. 2012;64(7):595-602.

31. Aaltonen J, et al. An autoimmune disease, APECED, caused by mutations in a novel gene featuring two PHD-type zinc-finger domains. Nat Genet. 1997;17(4):399-403.

32. Anderson MS, Su MA. Aire and T cell development. Curr Opin Immunol. 2011;23(2):198-206

33. Tusher VG, Tibshirani R, Chu G. Significance analysis of microarrays applied to the ionizing radiation response. Proc Natl Acad Sci U S A. 2001; 98(9):5116-5121.

34. Petri M. Hopkins Lupus cohort - 1999 update. Rheum Dis Clin North Am. 2000;26(2):199-213.

35. Wallace DJ, Hahn B, Dubois EL. Dubois' Lupus Erythematosus. Philadelphia, Pennsylvania, USA: Lippincott Williams and Wilkin; 2007.

36. Mackay F, Browning JL. BAFF: A fundamental survival factor for B cells. Nat Rev Immunol. 2002; 2(7):465-475.

37. Doreau A, et al. Interleukin 17 acts in synergy with B cell-activating factor to influence B cell biology and the pathophysiology of systemic lupus erythematosus. Nat Immunol. 2009;10(7):778-785.

38. Boneparth A, Davidson A. B-cell activating factor targeted therapy and lupus. Arthritis Res Ther. 2012; 14(suppl 4):S2.

39. Shum K, Askanase A. Belimumab and the clinical data. Curr Rheum Rep. 2012;14(4):310-317.

40. Bezalel S, Asher I, Elbirt D, Sthoeger ZM. Novel biological treatments for systemic lupus erythematosus: current and future modalities. Isr Med Assoc J. 2012; 14(8):508-514.

41. Baechler EC, et al. Interferon-inducible gene expression signature in peripheral blood cells of patients with severe lupus. Proc Natl Acad Sci U S A. 2003; 100(5):2610-2615.

42. Rönnblom L, Eloranta ML. The interferon signature in autoimmune diseases. Curr Opin Rheumatol. 2013; 25(2):248-253.

43. Bauer JW, et al. Interferon-regulated chemokines as biomarkers of systemic lupus erythematosus disease activity A validation study. Arthritis Rheum. 2009; 60(10):3098-3107.

44. La Cava A. Targeting the BLyS-APRIL signaling pathway in SLE. Clin Immunol. 2013;148(3):322-327.

45. van der Meide PH, Schellekens H. Anti-cytokine autoantibodies: epiphenomenon or critical modulators of cytokine action. Biotherapy. 1997;10(1):39-48.

46. Watanabe M, et al. Anti-cytokine autoantibodies are ubiquitous in healthy individuals. Febs Lett. 2007; 581(10):2017-2021.

47. Su LF, Kidd BA, Han A, Kotzin JJ, Davis MM. Virusspecific CD4(+) memory-phenotype $T$ cells are abundant in unexposed adults. Immunity. 2013; 38(2):373-383.

48. Okin D, Medzhitov R. Evolution of inflammatory diseases. Curr Biol. 2012;22(17):R733-R740.

49. Kattah MG, Alemi GR, Thibault DL, Balboni I, Utz PJ. A new two-color Fab labeling method for autoantigen protein microarrays. Nat Methods. 2006; 3(9):745-751.

50. Tabakman SM, et al. Plasmonic substrates for multiplexed protein microarrays with femtomolar sensitivity and broad dynamic range. Nat Commun. 2011; 2:466.

51. Armbruster DA, Pry T. Limit of blank, limit of detection and limit of quantitation. Clin Biochem Rev. 2008;29(suppl 1):S49-S52. 